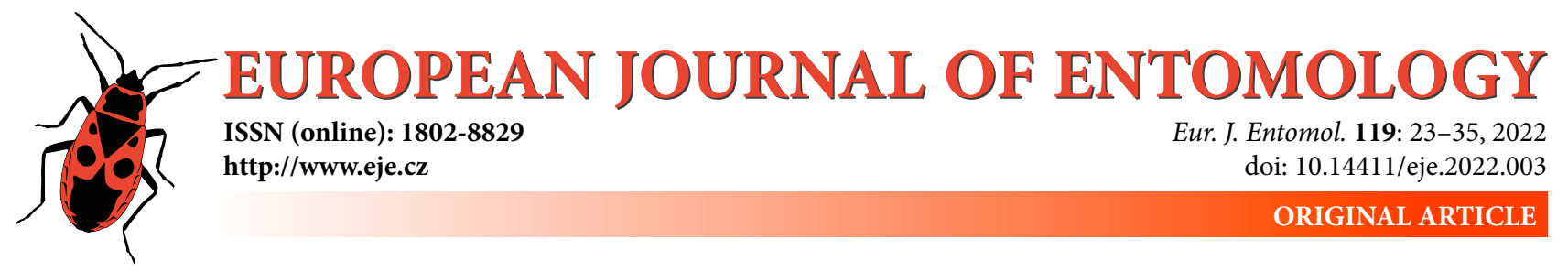

\title{
RNA interference-based characterization of Caspar, DREDD and FADD genes in immune signaling pathways of the red flour beetle, Tribolium castaneum (Coleoptera: Tenebrionidae)
}

\author{
KAKERU YOKOI ${ }^{1,2, *}$ (D), WATARU ITO ${ }^{1, *}$, DaIKI KATO ${ }^{1}$ and Ken MIURA ${ }^{1}$ \\ ${ }^{1}$ Applied Entomology Laboratory, Graduate School of Bioagricultural Sciences, Nagoya University, Furo-cho, Chikusa, \\ Nagoya 464-8601, Japan; e-mails: yokoi123@affrc.go.jp, wataru-ito@agr.nagoya-u.ac.jp, daiki-kato@agr.nagoya-u.ac.jp, \\ k-miura@agr.nagoya-u.ac.jp \\ ${ }^{2}$ Insect Design Technology Group, Division of Insect Advanced Technology, Institute of Agrobiological Sciences, NARO, Owashi, \\ Tsukuba 305-8643, Japan
}

Key words. Anti-microbial peptide, IMD pathway, Toll pathway

\begin{abstract}
We previously demonstrated that two immune signaling pathways, Toll and IMD, were concomitantly activated in the model beetle Tribolium castaneum by challenges to their immune system by several species of microbes, including Gram-positive and -negative bacteria as well as yeast. This contrasts with the Drosophila immune system in which more specific pathway activation depending on the type of microbe is well established. We suggest that the activation of an indiscriminate immune pathway in $T$. castaneum is due in part to an unselective recognition of pathogen-associated molecular patterns by the extracellular sensing modules of the two pathways. In order to obtain a more detailed understanding of the T. castaneum immune pathway, we investigated whether potential components of the T. castaneum IMD pathway, Caspar, DREDD and FADD, are involved in immune reactions triggered by microbial challenges. A sequence analysis of these three genes with the orthologues of other species, including insects, mouse and human, indicated that $T$. castaneum Caspar, DREDD and FADD functioned as immune signal transducers, which are usually induced by microbial challenges. However, these genes were not induced by microbial challenges. To establish whether these genes are involved in immune reactions, we used RNA interference-mediated knockdown of these genes to assess the microbial induction levels of the representative read-out antimicrobial peptide genes of the respective classes. The results indicated that these genes encode the canonical constituents of the IMD pathway of this beetle. DREDD and FADD influenced the induction of Toll-dependent antimicrobial peptide genes, providing novel crosstalk points between the two immune pathways, which appears to support indiscriminate pathway activation in T. castaneum. Furthermore, the phenotypes of DREDD or FADD knockdown pupae challenged by the two model bacterial pathogens correlated with AMP gene induction in the respective knockdowns, indicating that these intracellular factors contributed to antibacterial host defenses.
\end{abstract}

\section{INTRODUCTION}

The insect immune system is solely composed of innate immune reactions that utilize germ line-encoded receptors to detect the invasion of several distinct pathogens (Hultmark, 2003; Ferrandon et al., 2007; Lemaitre \& Hoffmann, 2007). When recognized, invading pathogens are attacked by a battery of innate immune responses. Insect immune responses as well as vertebrate immune responses may be conveniently divided into cellular and humoral immunities, the latter of which involves the systemic and robust production of antimicrobial peptides (AMPs) as a major and well-characterized response. Intensive studies on the model organism Drosophila melanogaster (Meigen) over the past two decades have delineated the paths and fac- tors that uphold the AMP production response as one of the hallmarks of insect immunity.

Extracellular pathogen recognition leads to the activation of two signaling pathways in Drosophila, the Toll and IMD pathways (Valanne et al., 2011; Kleino \& Silverman, 2014). The Toll pathway is mainly activated by either Lys-type peptidoglycan associated with most Grampositive bacteria or fungal $\beta-1,3$ glucan, and the recognition of these two pathogen-associated molecular patterns (PAMPs) in circulation is mainly executed by peptidoglycan recognition protein SA (PGRP-SA) and Gram-negative binding protein 3, respectively (Gobert et al., 2003; Gottar et al., 2006). PAMP binding to these receptors subsequently activates a downstream serine protease cascade, which leads to the proteolytic activation of the Toll ligand Spät-

\footnotetext{
* These authors equally contributed.
} 
zle (Spz) (Weber et al., 2003). Two Toll receptors, bound by two processed $\mathrm{Spz}$ dimers, undergo a conformational change and recruit the adaptor protein MyD88 through intracellular TID domains (Gangloff et al., 2008). This interaction also recruits another adaptor protein, Tube and the kinase Pelle via death domain (DD)-mediated interactions to form a heterotrimer complex comprising MyD88, Tube and Pelle (Sun et al., 2002). The formation of heterotrimers leads to the phosphorylation and degradation of Cactus, a Drosophila inhibitor of NF- $\mathrm{B}$, through paths that are not yet identified, and the NF- $\kappa$ B class transcription factor Dif or Dorsal translocates into the nucleus and activates a set of targets, including several AMP genes (Wu \& Anderson, 1998).

Gram-negative bacterial infections preferentially activate the IMD pathway (Kleino \& Silverman, 2014). Mesodiaminopimelic acid (DAP)-type peptidoglycan associated with Gram-negative bacteria and Gram-positive bacilli are mainly sensed by the membrane-bound receptor, PGRPLC (Choe et al., 2002, 2005). Ligand binding may promote the dimerization or multimerization of PGRP-LC, and its cytoplasmic portion, harbouring the receptor-interacting protein homeotypic interaction motif, interacts with the adaptor protein IMD (Kaneko et al., 2006). IMD recruits the initiator caspase- 8 homolog DREDD via the adaptor function of FADD (Leulier et al., 2000; Naitza et al., 2002), and DREDD is activated by mechanisms that have yet to be completely defined, but include K63 polyubiquitination by the E3 ubiquitin ligase DIAP2 (Meinander et al., 2012). Activated DREDD cleaves IMD at $\mathrm{Asp}^{30}$, which exposes the interaction interface to DIAP2, and cleaved IMD is also subjected to K63 ubiquitination (Paquette et al., 2010). The polyubiquitin chain recruits and activates the kinase TAK1, which, in turn, activates the JNK and IKK branches of the IMD pathway (Rutschmann et al., 2000; Silverman et al., 2000, 2003). The Drosophila IKK complex comprises catalytic IKK $\beta$ and regulatory IKK $\gamma$ subunits. The IKK complex is thought to phosphorylate another NF- $\mathrm{KB}$ class transcription factor, Relish (Stöven et al., 2000; Stöven et al., 2003). Phosphorylated Relish is then translocated into the nucleus after the proteolytic removal of its ankyrin portion by DREDD, ultimately activating its target genes.

While Drosophila immune signaling events leading to the activation of humoral responses have been examined in detail, comprehensive information on other insect species, particularly non-dipteran insects, is limited. This may be attributed to the unavailability of genomic information, except for some model insects, and also to the incompatibility of genetic manipulation techniques, including RNA interference (RNAi) in some experimental systems. Therefore, some of the information obtained on immune responses in non-Drosophila insects may be annotated on the basis of the Drosophila framework.

We started research on insect immune signaling using the model beetle Tribolium castaneum (Herbst) because information on its genome is available and this beetle exhibits systemic RNAi responses, and our aim was to provide another framework for an insect immune system
(Tomoyasu et al., 2008; Tribolium Genome Sequencing Consortium, 2008). In the initial phase, we characterized all nine AMP genes in the genome of $T$. castaneum and categorized them into IMD pathway-dependent group I, Toll pathway-dependent group III, and co-dependent group II as well as non-inducible group IV genes by utilizing the RNAi of the pathway-specific intracellular adaptor protein genes, IMD and MyD88 (Yokoi et al., 2012b). We subsequently demonstrated that epistatic relationships between the pathways and subclasses of NF- $\kappa \mathrm{B}$ transcription factors in Drosophila were also present in T. castaneum (Yokoi et al., 2012a). Collectively, these findings indicated that the Toll and IMD pathways exist in this beetle, and we also noted that the specificity of pathway activation was distinct from that in Drosophila because the target AMP genes of both pathways were concomitantly activated by any of the five microbial species used, including Gramnegative and -positive bacteria as well as budding yeast. We considered this indiscriminate pathway activation to be a distinct feature of $T$. castaneum immune signaling, which differs from that of Drosophila, and we have been investigating the molecular mechanisms that support this phenomenon. We demonstrated that $P G R P-L A$ encodes the canonical receptor of the IMD pathway in this beetle, and also that this isoform recognizes both Gram-positive and -negative bacteria (Koyama et al., 2015). The finding of extracellular pathogen recognition may account in part for the aforementioned immune pathway activation with lower specificity. In the present study, to extend our understanding of the $T$. castaneum immune system, we focused on potential receptor-proximal intracellular components in the IMD pathways of T. castaneum, DREDD and FADD, as well as the DREDD inhibitor Caspar (Kim et al., 2006), and investigated whether these factors provide bifurcating points of immune signaling and are involved in immune reactions against bacterial infections.

\section{MATERIALS AND METHODS}

\section{Insect rearing}

T. castaneum was reared on whole wheat flour at $30^{\circ} \mathrm{C}$ in the dark and their pupae were prepared as previously described (Yokoi et al., 2012b).

\section{Microbes and injections into insects}

Escherichia coli DH5a, Micrococcus luteus ATCC4698 and the budding yeast, Saccharomyces cerevisiae S288C were used as heat-killed cell suspensions with defined O.D. values, as described in Yokoi et al. (2012a). Prepared suspensions of E. coli, $M$. luteus and S. cerevisiae were $2.9 \times 10^{8}, 2.9 \times 10^{7}$ and $5 \times 10^{6}$ cells/ml, respectively. Fifty nanolitres of heat-killed suspensions was injected into naïve or double strand RNA (dsRNA)-treated day 3 pupae using Nanoject II (Drummond Scientific Company) to elicit immune responses. Suspensions of live Enterobacter cloacae and Bacillus subtilis were also prepared and used in survival assays, as previously described in Yokoi et al. (2012b). E. cloacae and B. subtilis were generous gifts from Dr. Y. Yagi of Nagoya University. S. cerevisiae S288C was from Dr. T. Ushimaru of Shizuoka University. M. luteus ATCC4698 was provided by the RIKEN Bioresource Center in Japan. 


\section{Genes examined in the present study}

The following T. castaneum gene sequences from reference genome version Tcas 2.0 were retrieved from Tcas2.0/annotation/ TCGleanPrediction data (URL: ftp://ftp.hgsc.bcm.edu/Tcastaneum/) available at "BCM-HGSC data" in the "Red Flour Beetle Genome Project" site (URL: https://www.hgsc.bcm.edu/arthropods/red-flour-beetle-genome-project) and utilized in the present study (Zou et al., 2007; Tribolium Genome Sequencing Consortium, 2008): Caspar (Gene ID in gene set data: GLEAN_09985); DREDD (GLEAN 14026); FADD (GLEAN_14042); Attacin 1 (Att1) (GLEAN_07737); (GLEAN_07739); Cecropin 2 (Cec2) (GLEAN_00499); Cecropin 3 (Cec3) (GLEAN_00500); Coleoptericin 1 (Col1) (GLEAN 05093 and GLEAN_10517: Col1 was split into two genes in gene set data.); Defensin 3 (Def3) (GLEAN_12469); IMD (GLEAN_10851); and the normalizer gene for qRT-PCR analyses, ribosomal protein L32 (RPL32) (GLEAN_06106).

\section{Real-time quantitative RT-PCR (qRT-PCR)}

Total RNA was extracted from the whole body of $T$. castaneum pupae using TRIZOL reagent (Invitrogen). After spectrophotometric scanning and assessments, $0.5 \mu \mathrm{g}$ of total RNA was converted to first-strand cDNA utilizing a PrimeScript RT Reagent Kit with gDNA Eraser (TAKARA), and first-strand cDNA was analysed by qRT-PCR using the SYBR Premix Ex Taq Perfect Real Time Kit Tli RNAaseH Plus (TAKARA) and Thermal Cycler Dice Real Time System (Model TP800, TAKARA). The sequences of primer pairs used for qRT-PCR of Caspar, DREDD and FADD are shown in the Supplementary Table 1 . The sequences of qRT-PCR primers for the other genes and PCR conditions are described in our previous studies (Yokoi et al., 2012a, b).

\section{RNAi}

RNAi-mediated gene knockdown was done as described in our previous studies (Yokoi et al., 2012a, b). Briefly, T7 RNA polymerase promoter sequence-tagged cDNA generated by conventional PCR was used as a template for dsRNA synthesis with the MEGAscript RNAi Kit (Ambion). One hundred nanograms of dsRNA, typically in a volume of $33 \mathrm{nl}$, for each target gene was injected into day 0 pupae using Nanoject II. Pupae were examined $72 \mathrm{~h}$ later for knockdown efficiency or challenged with microbes. dsRNA with a partial maltose binding protein $E$ ( malE) sequence served as a negative control. The sequences of primer pairs used for the production of T7 RNA polymerase promoter sequence (5'-TAATACGACTCACTATAGGG-3')-tagged cDNA is shown in Supplementary Table 1. The sequences of primer pairs used for IMD and malE cDNA template preparation are described in Yokoi et al. (2012b).

\section{Survival assay}

Day 0 pupae were treated with dsRNA. Pupae were challenged $72 \mathrm{~h}$ after the dsRNA treatment with live E. cloacae $\left(50 \mathrm{nl}, \mathrm{A}_{600}=\right.$ $0.1)$ or B. subtilis (100 nl, $\left.\mathrm{A}_{600}=3.0\right)$, as described in Yokoi et al. (2012a). The survival of pupae was thereafter recorded every 24 h. Data were presented as Kaplan-Meier plots and $P$-values calculated using the Gehan-Breslow-Wilcoxon test by using a software package (Ekuseru-Toukei 2010 ver. 1.13, Social Survey Research Information Co., Ltd.).

\section{Sequence analyses}

Multiple amino acid sequence alignments were performed using Clustal Omega (version 1.2.4) (Sievers \& Higgins, 2021). Newick files were constructed from Clustal Omega output files (aln files) using FastTree program version 2.1.10 (Price et al., 2009), and approximate maximum-likelihood phylogenetic trees constructed using newick files with MEGAX version 10.1.7
(Kumar et al., 2018). All sequence analyses were done with default settings. To search for functional domains in the Caspar, DREDD and FADD of T. castaneum, the predicted amino acid sequences of these genes were analysed using InterProScan with InterPro version 86.0 (Blum et al., 2021, URL: https://www.ebi. ac.uk/interpro/search/sequence/). All raw data from these analyses, which are fasta, aln, newick and gff files, are available in figshare (see Supplementary Data 1-3).

\section{RESULTS}

\section{Phylogenetic analyses of Caspar, DREDD and FADD, and search for functional domains in $T$. castaneum Caspar, DREDD and FADD}

The sequences of T. castaneum orthologues for Caspar, DREDD and FADD (Tc-Caspar, Tc-DREDD, and Tc-FADD, respectively) were obtained from gene set data based on T. castaneum genome data (Zou et al., 2007; Tribolium Genome Sequencing Consortium, 2008), and their deduced amino acid sequences were subjected to phylogenetic analyses (Supplementary Fig. 1) together with the related sequences of other organisms [Aedes aegypti (Linnaeus), Anopheles gambiae (Giles), D. melanogaster, Bombyx mori (Linnaeus), Apis mellifera (Linnaeus), Nilaparvata lugens (Stål), mouse (Linnaeus) and human] (Supplementary Fig. 1). Furthermore, functional domains in the predicted amino acid sequences of Tc-Caspar, Tc-DREDD and Tc-FADD were searched for by InterProScan (Supplementary Fig. 2). Supplementary Fig. 1A shows the phylogenetic tree of Caspar-related proteins. Tc-Caspar forms a clade with B. mori Caspar (Bm-Caspar), indicating that Bm-Caspar is the closest protein to Tc-Caspar in the phylogenetic tree. The results of InterProScan showed that Tc-Caspar has functionally important domains, which are the FAF1_UBA domain (functions as a Fas-associated domain), ubiquitin-associated (UAS) domain and ubiquitinlike regulatory X domain (UBX) (Supplementary Fig. 2A). The death effecter domain-interacting domain (DEDID) is not predicted but the ubiquitin-like motif is predicted (IPR029071) (Chu et al., 1995; Park et al., 2004; Kim et al., 2006; He et al., 2013). However, the Ubiquitin-like motif is found in the Ras-binding domain, suggesting that this motif functions as DEDID (Nassar et al., 1995). Similar analyses were performed on DREDD/Caspase 8 proteins and FADD proteins (the FADD orthologue is not present in the $N$. lugens genome). In the phylogenetic trees of $D R E D D$ and FADD, Tc-DREDD is located near a clade including $D$. melanogaster, A. aegypti and A. gambiae DEREDDs (Supplementary Fig. 1B), while Tc-FADD is located in a clade comprising D. melanogaster and B. mori FADDs (Supplementary Fig. 1C). The results of domain searches in Tc-DREDD InterProScan showed that Tc-DREDD has two caspase domains and a single death effector domain (DED) (Supplementary Fig. 2B), which were previously reported in D. melanogaster DEREDD and shown to be functionally important (Luelier et al., 2000). Tc-FADD has a single death domain and another death domain in the homologous superfamily result, which might correspond to the death domain and death -interacting domains, respectively in D. melanogaster FADD (Supplementary Fig. 2C) 

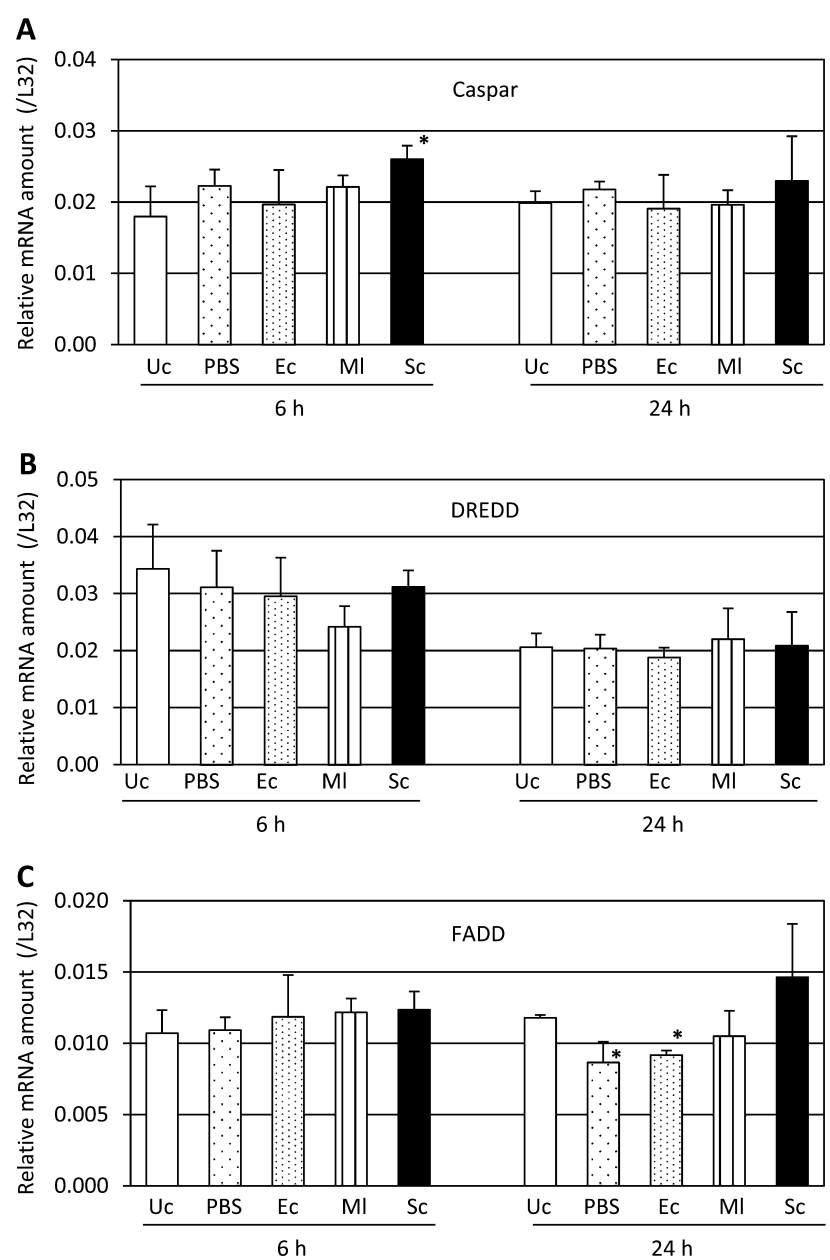

Fig. 1. Microbial challenge and effects on mRNA levels of Caspar, $D R E D D$ and $F A D D$. Day 3 pupae were challenged with a heatkilled cell suspension of $E$. coli (Ec), M. luteus (MI) or $S$. cerevisiae (Sc) and the mRNA levels of Caspar (A), DREDD (B) and FADD (C) were assessed using qRT-PCR, 6 and $24 \mathrm{~h}$ after a challenge. Unchallenged (Uc) and PBS-injected day 3 pupae (PBS) served as controls. mRNA values are shown relative to RpL32 levels in the same samples. Experiments were independently carried out at least three times with 3 to 4 animals, and each column represents the mean \pm S.D. Asterisks indicate a significant difference $(P<0.05$ based on Student's $t$-tests) from the corresponding Uc controls.

(Hu \& Yang, 2000). These results suggest that Caspar, DREDD and FADD in T. castaneum encode the functional counterparts of proteins in other species.

\section{Microbial induction of Caspar, DREDD and FADD}

The levels of expression of insect immune-related genes are frequently influenced by microbial invasion in several organisms, such as, D. melanogaster and T. castaneum (De Gregorio et al., 2001, 2002; Yokoi et al., 2012a, b). To confirm these findings, we investigated whether $T$. castaneum Caspar, DREDD and FADD were induced by an injection of heat-killed microbes (Fig. 1). The results indicate that the mRNA amounts of Caspar (Fig. 1A), DREDD (Fig. $1 \mathrm{~B})$ and $F A D D$ (Fig. 1C) were not or only weakly affected 6 and $24 \mathrm{~h}$ after the challenges with $E$. coli, M. luteus and S. cerevisiae.

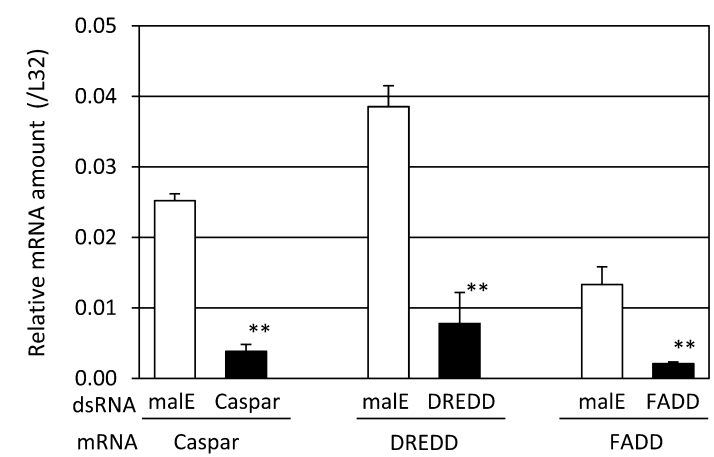

Fig. 2. Efficiency evaluation of RNAi-mediated gene knockdown. Day 0 pupa were injected with $100 \mathrm{ng}$ of Caspar, DREDD, FADD or control malE dsRNA, and the levels of mRNA relative to those of $R p L 32$ were measured $72 \mathrm{~h}$ later. Experiments were independently carried out at least three times, and the mean \pm S.D is shown. Double asterisks indicate $P<0.01$ versus controls based on Student's $t$-test.

\section{Efficiency of the injection of dsRNA in the knockdown of Caspar, DREDD and FADD}

Prior to the functional assay of these three genes, we determined whether they were effectively knockdown by RNAi. Day 0 pupae were injected with the dsRNA for the respective targets along with control malE dsRNA and the amounts of mRNA were measured $72 \mathrm{~h}$ later. The results are presented in Fig. 2, which show that gene knockdown resulted in 80 to $85 \%$ reductions in the amounts of mRNA for all three genes.

\section{Effects of the knockdown of Caspar, DREDD and $F A D D$ on the microbial induction of group I AMP genes}

Using Caspar, DREDD and FADD knockdown pupae, the effects of the knockdown of these genes on the expressions of the group I genes, Att1 (Fig. 3) and Coll (Fig. 4), are largely dependent on the IMD pathway (Yokoi et al., 2012b). Knockdown pupae and pupae treated with malE dsRNA as controls were challenged with the heatkilled cells of E. coli, M. luteus or S. cerevisiae, and the levels of mRNA in these treated pupae were measured 6 and $24 \mathrm{~h}$ after the challenges. As previously reported the amounts of Att1 and Coll mRNA in unchallenged pupae were approximately 0.01 and 0.05 respectively (Yokoi et al., 2012a), which are clearly lower than those in malE dsRNA-treated control pupae, 6 and $24 \mathrm{~h}$, post challenge with three microbes, except for Att1 and Coll post challenge 24 h by $S$. cerevisiae. Thus, the results in Figs 3 and 4 can be considered to show the effects of the knockdown of Caspar, DREDD and FADD on Coll inductions by the three microbial challenges, except for Fig. 4F. The mRNA amounts of Attl in DREDD, FADD and Caspar dsRNA pre-treated pupae $6 \mathrm{~h}$ after the $E$. coli challenge were not significantly different from those in control pupae, whereas they were significantly lower in $D R E D D$ and FADD knockdown pupae than in control pupae $24 \mathrm{~h}$ after a challenge (Fig. 3A and 3B). Pupae treated with Caspar dsRNA, 6 and $24 \mathrm{~h}$ after the $E$. coli challenge had slightly higher mRNA amounts of Attl than control pupae. Comparisons 

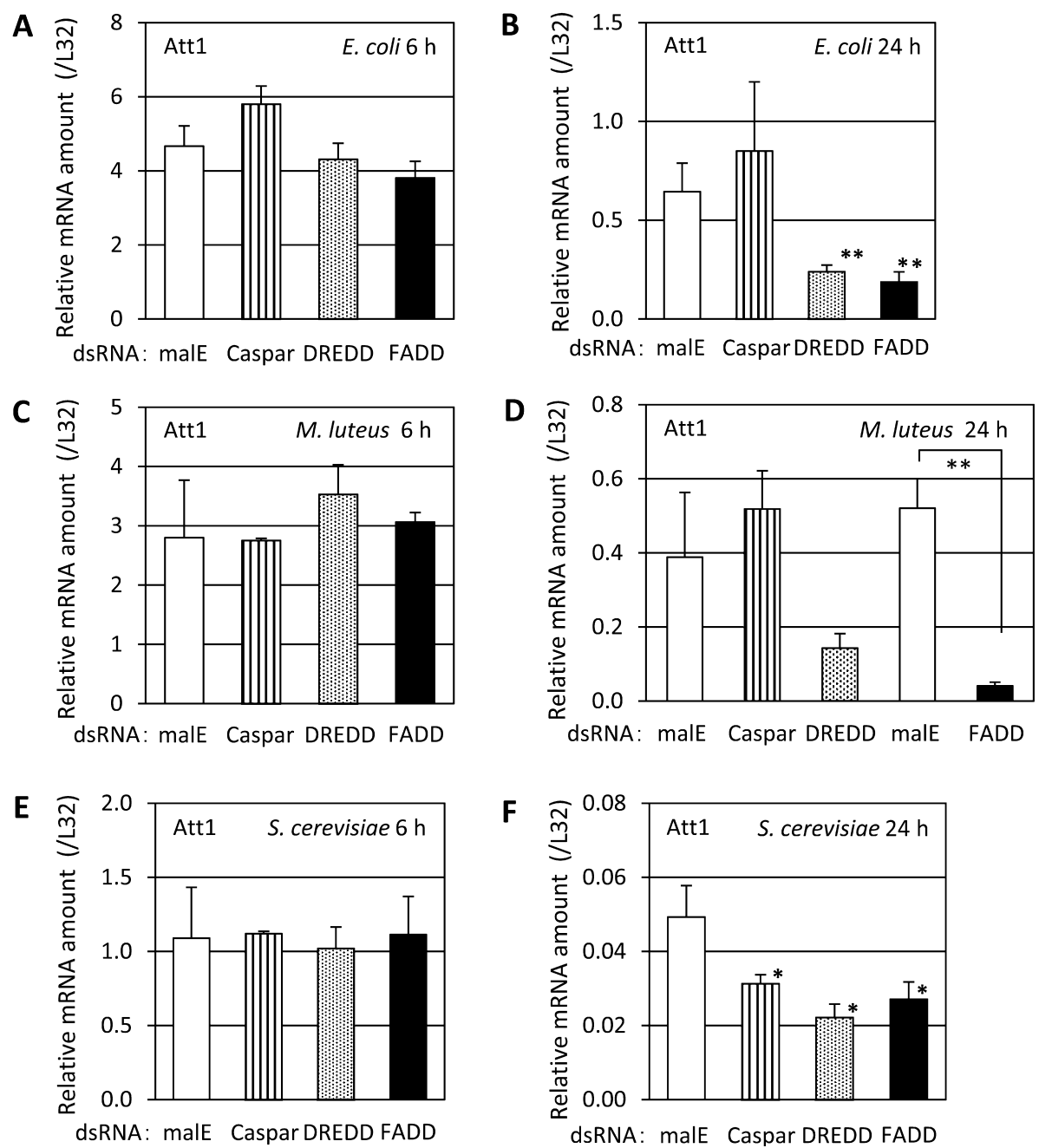

Fig. 3. Effects of the knockdown of Caspar, DREDD and FADD on the expression of the group I AMP gene, Att1, in pupae challenged by a microbe. Day 0 pupae were injected with a preparation of dsRNA. Seventy-two hours later, the dsRNA-treated pupae were injected with a heat-killed cell suspension of $E$. coli (A and B), M. luteus (C and D) or S. cerevisiae (E and F). The relative levels of mRNA of Att1 to $R p L 32$ were assessed $6(A, C$ and $E)$ and $24 \mathrm{~h}(\mathrm{~B}, \mathrm{D}$ and $\mathrm{E})$ after the microbial challenge and are shown as the mean \pm S.D. Experiments were independently repeated at least three times with three animals each. Single asterisks indicate $P<0.05$ versus malE controls based on Student's $t$-tests, while double asterisks denote $P<0.01$.

of the mRNA amounts of Att1 in control pupae challenged with $E$. coli and M. luteus revealed that the levels of activation of Attl induced by the M. luteus challenge were lower than those of the $E$. coli challenge, which is consistent with previous findings (Yokoi et al., 2012a). The effects of the knockdown of DREDD, FADD, or Caspar on gene induction did not significantly alter the levels of Att 1 mRNA $6 \mathrm{~h}$ after the $M$. luteus challenge (Fig. 3C). Pupae treated with DREDD or FADD dsRNA had lower mRNA levels of Att1 $24 \mathrm{~h}$ after the M. luteus challenge than control pupae (Att1 mRNA levels of DREDD knockdown and control pupae were clearly different but not significantly so), while the pupae with Caspar dsRNA showed modest enhancements $24 \mathrm{~h}$ after the M. luteus challenge (Fig. 3D). Six hours after the $S$. cerevisiae challenge, no significant differences were observed in Att1 mRNA levels between any dsRNA-treated and control pupae (Fig. 3E). Significantly lower mRNA levels of Att1 $24 \mathrm{~h}$ after the $S$. cerevisiae challenge were recorded in DREDD, FADD and Caspar dsRNA-treated pupae than in control pupae (Fig. 3F).
Similar knockdown and microbial induction experiments were performed for the group I gene, Coll (Fig. 4). Overall results were similar to those for Att1. Six hours after the E. coli challenge, Col1 mRNA amounts were significantly lower in $D R E D D$ knockdown pupae than in control pupae, but did not differ significantly between FADD knockdown and control pupae (Fig. 4A). Coll mRNA amounts were significantly higher in Caspar knockdown pupae than in control pupae. Twenty-four hours after the $E$. coli challenge, Coll mRNA amounts were significantly lower in DREDD and FADD knockdown pupae than in control pupae, while those in Caspar knockdown pupae were slightly higher (Fig. 4B). Coll mRNA amounts in DREDD, FADD and Caspar knockdown pupae $6 \mathrm{~h}$ after the $M$. luteus challenge did not differ significantly from those in the control pupae (Fig. 4C). Twenty-four hours after the M. luteus challenge, Coll mRNA amounts were slightly higher and lower in Caspar and DREDD knockdown pupae, respectively, than in control pupae, but were significantly lower in $F A D D$ knockdown pupae than in 
A

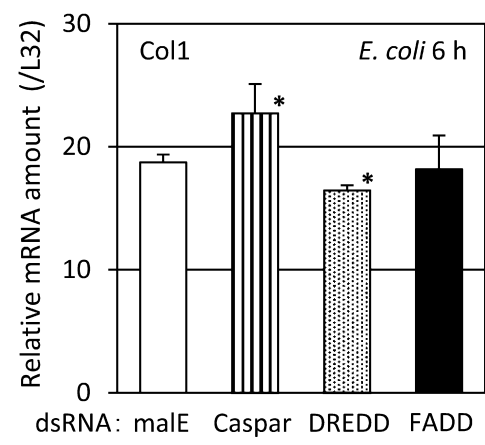

C

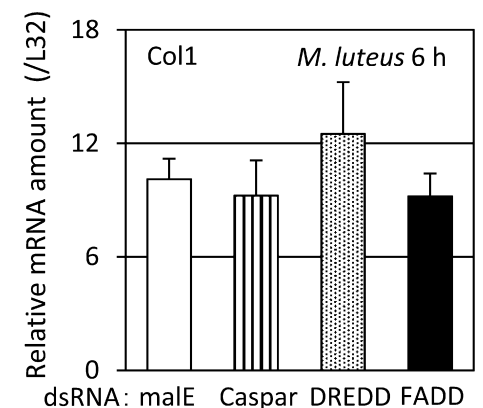

E

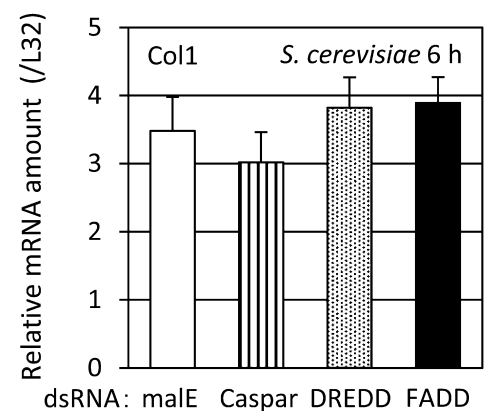

B

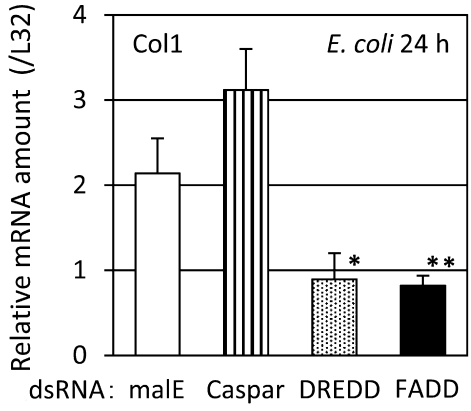

D

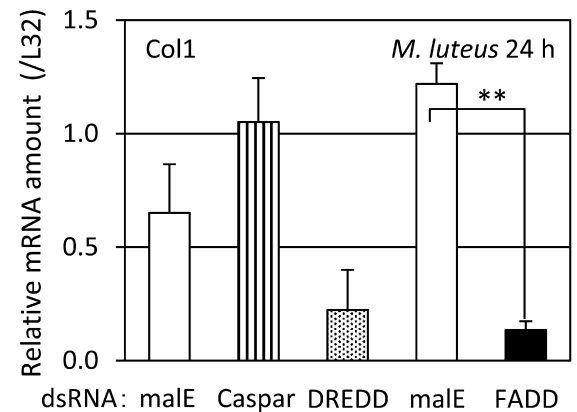

F

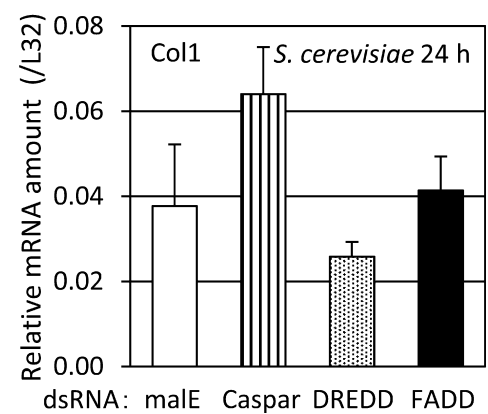

Fig. 4. Effects of the knockdown of Caspar, DREDD and FADD on the expression of the group I AMP gene, Col1, in pupae challenged by a microbe. Day 0 pupae were injected with preparations of dsRNA. Seventy-two hours later, the dsRNA-treated pupae were injected with a heat-killed cell suspension of $E$. coli $(A$ and $B)$, M. luteus (C and D) or S. cerevisiae (E and $F)$. The relative levels of mRNA of Att1 to $R p L 32$ were assessed $6(A, C$ and $E)$ and $24 \mathrm{~h}(\mathrm{~B}, \mathrm{D}$ and $\mathrm{E})$ after a microbial challenge and shown as the mean \pm S.D. Experiments were independently repeated at least three times with three animals each. Single asterisks indicate $P<0.05$ versus malE controls based on Student's $t$-tests, while double asterisks denote $P<0.01$.

control pupae (Fig. 4D). Similar to $6 \mathrm{~h}$ after the M. luteus challenge, no significant differences were recorded in Coll mRNA amounts between DREDD, FADD and Caspar knockdown pupae and control pupae $6 \mathrm{~h}$ after the $S$. cerevisiae challenge (Fig. 4E). Coll mRNA amounts varied among DREDD, FADD and Caspar knockdown pupae and control pupae $24 \mathrm{~h}$ after the $S$. cerevisiae challenge, but were not significantly different (Fig. 4F).

\section{Effects of the knockdown of Caspar, DREDD and $F A D D$ on the microbial induction of the group II AMP gene}

The results recorded for the group II gene Def3 were distinct from those for group I genes (Fig. 5). The level of expression of $\operatorname{Def} 3$ in unchallenged pupae is approximately 0.003 (Yokoi et al., 2012a), which is clearly a lower value than that recorded in pupae treated with malE dsRNA and post challenged 6 and $24 \mathrm{~h}$ later with three microbes, except for post challenge $24 \mathrm{~h}$ with $M$. luteus and $S$. cerevisiae. Thus, results in Fig. 5A, B, C and E are considered to show effects of the knockdown of Caspar, DREDD and FADD on Def3 inductions by the three microbial challenges. Levels of Def 3 expression $6 \mathrm{~h}$ after the $E$. coli challenge were significantly lower in $D R E D D$ knockdown pupae than in control pupae, while those in Caspar knockdown pupae were significantly higher (Fig. 5A). Levels of expression of Def3 were slightly lower in FADD knockdown pupae. Similar results were obtained for pupae $24 \mathrm{~h}$ after the $E$. coli challenge (Fig. 5B). Levels of expression of Def3 were similar in pupae $6 \mathrm{~h}$ after the M. luteus and E. coli challenges, with the lack of significant differences attributed to large variations in malE control values (Fig. 5C). Twentyfour hours after the $M$. luteus challenge, the levels of expression of Def3 were significantly lower in DREDD and $F A D D$ knockdown pupae than in control pupae, but were higher in Caspar knockdown pupae (Fig. 5D). Regarding the induction by $S$. cerevisiae $6 \mathrm{~h}$ after the challenge, the knockdown of DREDD and FADD appeared to suppress the induction of Def3 (Fig. 5E). The induction profiles of Def3 by $M$. luteus and $S$. cerevisiae at $24 \mathrm{~h}$ post challenge 
A

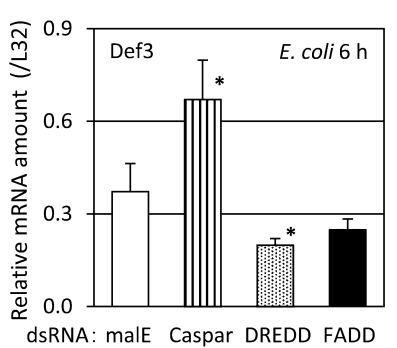

C

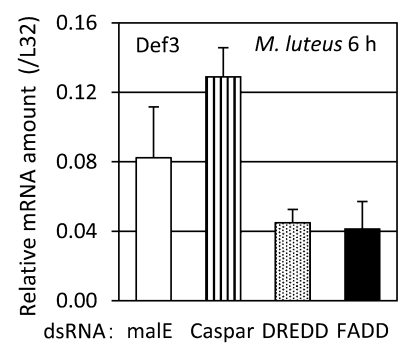

E

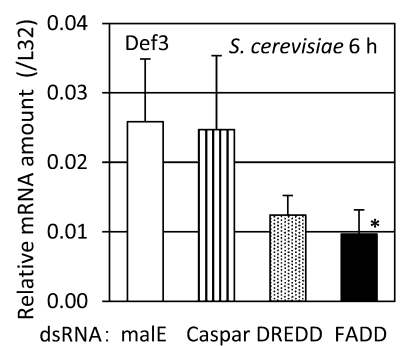

B

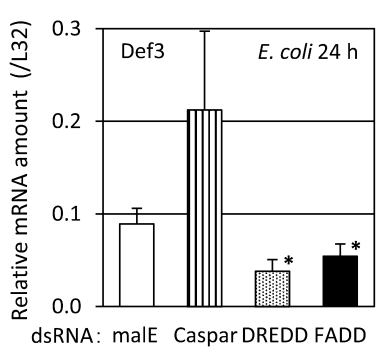

D

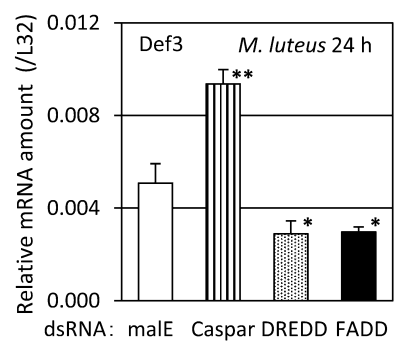

$\mathbf{F}$

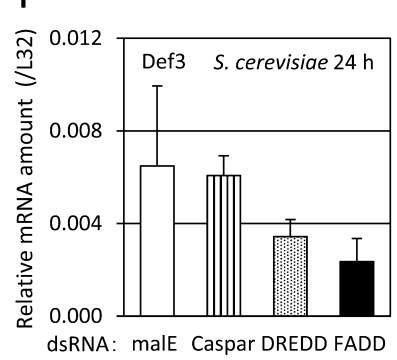

Fig. 5. Effects of the knockdown of Caspar, DREDD and FADD on the expressions of the group II AMP gene, Def3, in pupae challenged by a microbe. Day 0 pupae were injected with preparations of dsRNA. Seventy-two hours later, the dsRNA-treated pupae were injected with a heat-killed cell suspension of $E$. coli (A and B), $M$. luteus ( $C$ and $D$ ) or $S$. cerevisiae (E and $F)$. The relative levels of mRNA of Att 1 to RpL32 were assessed 6 (A, C and E) and $24 \mathrm{~h}$ $(B, D$ and $F$ ) after the microbial challenge and shown as the mean \pm S.D. Experiments were independently repeated at least three times with three animals each. Single asterisks indicate $P<0.05$ versus malE controls based on Student's t-tests.

apparently exhibits some knockdown effects, but in both cases the induction will have ceased by this time according to our previous results (Yokoi et al., 2012a, b) and is well supported by the low Def3 mRNA levels (Fig. 5D and 5F).

\section{Effects of the knockdown of Caspar, DREDD and $F A D D$ on the microbial induction of group III AMP genes}

Two group III genes, $\mathrm{Cec} 2$ and $\mathrm{Cec} 3$, the expression of which is largely dependent on Toll signaling, were investigated (Figs 6 and 7) (Yokoi et al., 2012b). the values of the expressions of $\mathrm{Cec} 2$ and $\mathrm{Cec} 3$ in unchallenged pupae is approximately 0.02 and 0.1 , respectively (Yokoi et al., 2012a, b), which are clearly lower than those recorded for malE dsRNA-treated controls depicted in Figs 6 and 7. Thus, the results in Figs 6 and 7 can be considered to show the effects of the knockdown of Caspar, DREDD and FADD on the induction of $\mathrm{Cec} 2$ and $\mathrm{Cec} 3$, respectively, by the three

\section{A}

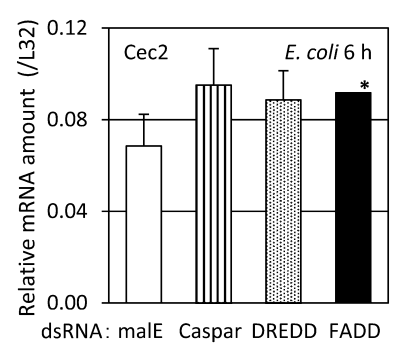

B

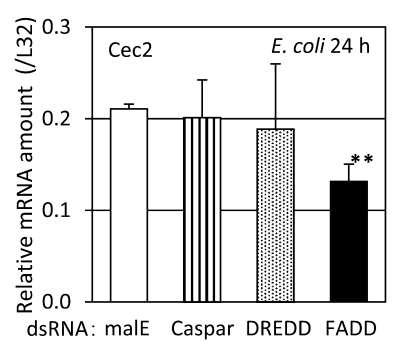

C

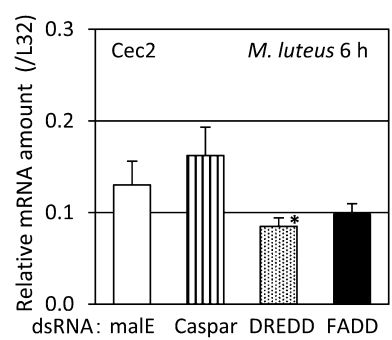

D

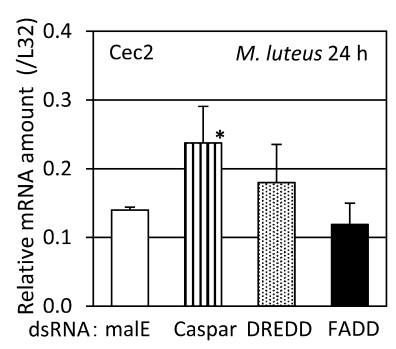

$\mathbf{E}$

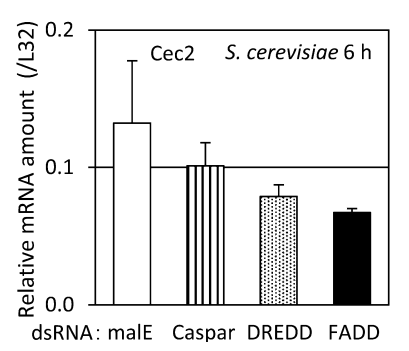

$\mathbf{F}$

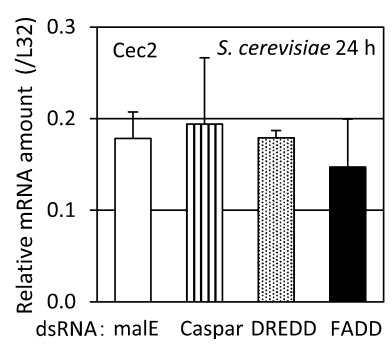

Fig. 6. Effects of the knockdown of Caspar, DREDD and FADD on the expression of the group III AMP gene, Cec2, in pupae challenged by a microbe. Day 0 pupae were injected with preparations of dsRNA. Seventy-two hours later, the dsRNA-treated pupae were injected with a heat-killed cell suspension of $E$. coli (A and $B), M$. luteus $(C$ and $D)$ or $S$. cerevisiae ( $E$ and $F$ ). The relative levels of mRNA of Att1 to RpL32 were assessed 6 (A, C and E) and $24 \mathrm{~h}$ (B, $\mathrm{D}$ and $\mathrm{F}$ ) after a microbial challenge and shown as the mean \pm S.D. Experiments were independently repeated at least three times with three animals each. Single asterisks indicate $P<0.05$ versus malE controls based on Student's $t$-tests.

microbial challenges. The level of expression of $\mathrm{Cec} 2$ in Caspar and DREDD knockdown pupae $6 \mathrm{~h}$ after the challenge with $E$. coli did not differ significantly from those in control pupae, while modest up-regulation and suppression were only recorded in FADD knockdown pupae 6 and $24 \mathrm{~h}$, respectively, after the challenge (Fig. 6A and 6B). Six hours after the challenge with M. luteus, the amounts of Cec2 mRNA were significantly and slightly lower in $D R E D D$ and $F A D D$ knockdown pupae, respectively, than in control pupae (Fig. 6C). Twenty-four hours after the challenge, the amounts of Cec2 mRNA in DREDD and $F A D D$ knockdown pupae did not differ significantly from those in control pupae (Fig. 6D). The knockdown of Caspar appeared to activate effects that became more prominent (also significantly different) in the later phase of induction (Fig. 6C and 6D). No significant differences were recorded between Caspar, DREDD and FADD knockdown 
A
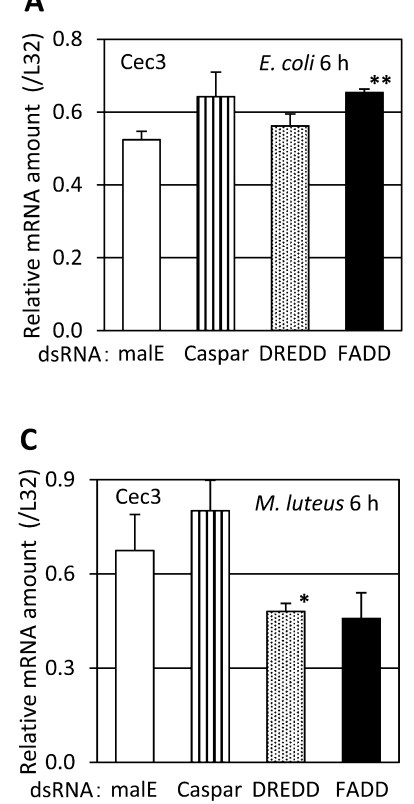

E

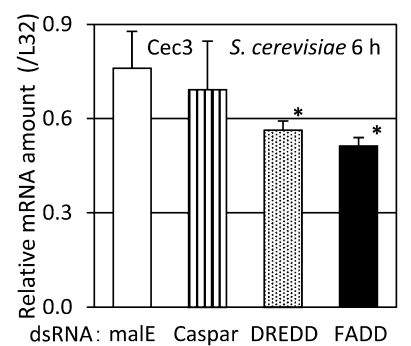

B

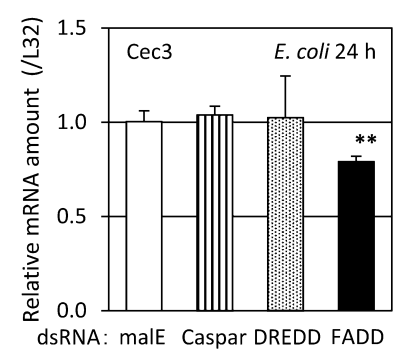

D

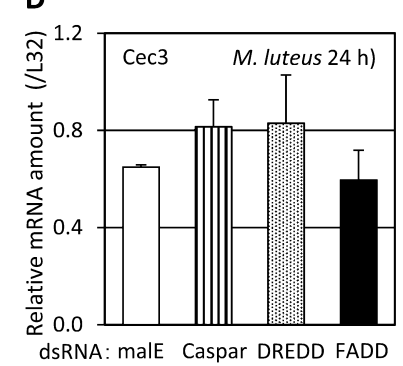

$\mathbf{F}$

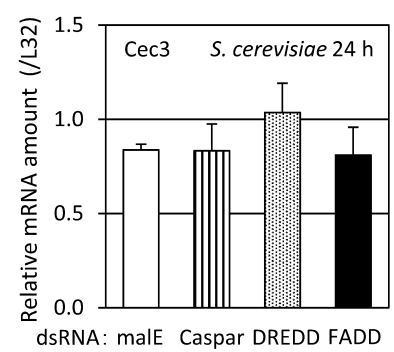

Fig. 7. Effects of the knockdown of Caspar, DREDD and FADD on the expression of the group III AMP gene, $\mathrm{Cec} 3$, in pupae challenged by a microbe. Day 0 pupae were injected with preparations of dsRNA. Seventy-two hours later, the dsRNA-treated pupae were injected with a heat-killed cell suspension of $E$. coli (A and B), $M$. luteus (C and D) or $S$. cerevisiae (E and $F)$. The relative levels of mRNA of Att1 to RpL32 were assessed 6 (A, C and E) and $24 \mathrm{~h}$ (B, $\mathrm{D}$ and $\mathrm{F}$ ) after a microbial challenge and shown as the mean \pm S.D. Experiments were independently repeated at least three times with three animals each. Single asterisks indicate $P<0.05$ versus malE controls based on Student's $t$-tests.

pupae and control pupae 6 or $24 \mathrm{~h}$ after the challenge with S. cerevisiae (Fig. 6E and 6F).

Levels of expression of $\mathrm{Cec} 36 \mathrm{~h}$ after the challenge with E. coli were slightly higher in Caspar and FADD knockdown pupae than in control pupae, but were similar in DREDD knockdown pupae and control pupae (Fig. 7A). Twenty-four hours after the challenge, the levels of expression of $\mathrm{Cec} 3$ were slightly lower in FADD knockdown pupae than in control pupae, while there was no difference of the levels of $\mathrm{Cec} 3$ expression between Caspar or FADD knockdown and control pupae (Fig. 7B). Six hours after the challenge with $M$. luteus, amounts of $\mathrm{Cec} 3 \mathrm{mRNA}$ in $D R E D D$ and FADD knockdown pupae were less than in the controls (Fig. 7C). Amounts of $\mathrm{Cec} 36 \mathrm{~h}$ after the challenge with $M$. luteus were slightly more abundant in Caspar knockdown pupae. Twenty-four hours after the M. luteus challenge, no significant differences were recorded in the

amounts of Cec3 mRNA in Caspar, DREDD and FADD knockdown pupae and control pupae (Fig. 7D). Furthermore, amounts of $\mathrm{Cec} 3$ mRNA did not differ significantly in Caspar knockdown and control pupae 6 or $24 \mathrm{~h}$ after the challenge with $S$. cerevisiae (Fig. $7 \mathrm{E}$ and $7 \mathrm{~F}$ ). Levels of expression of Cec3 $6 \mathrm{~h}$ after the challenge with $S$. cerevisiae were lower in DREDD and FADD knockdown pupae than in control pupae, while no significant differences were recorded $24 \mathrm{~h}$ after the challenge (Fig. 7E and 7F).

\section{Survival assays}

To clarify the involvement of these IMD pathwayassociated intracellular factors in the antimicrobial host defenses of T. castaneum, survival was assayed using two model pathogenic bacterial strains. Pupae pre-treated with dsRNA were inoculated intrahaemocoelically with defined doses of live E. cloacae or B. subtilis, and percentage survival was then recorded every 24 h. malE dsRNA-treated pupae served as negative controls, while those treated with $I M D$ dsRNA were positive controls. Results are shown in Kaplan-Meier plots (Fig. 8). In comparisons with the negative control, the knockdown of IMD severely impaired host defenses against E. cloacae and B. subtilis (Fig. 8A to 8D). $D R E D D$ and FADD knockdown pupae represented intermediate phenotypes. The knockdown of FADD had similar effects to that of IMD in terms of defense against the two bacteria, while those of the knockdown of DREDD appeared to be weaker. DREDD knockdown pupae succumbed to E. cloacae infection slightly faster than malE controls $(P=0.0536$, Fig. 8B). In addition, the knockdown of DREDD significantly weakened host defense against $B$. subtilis, and the $P$-value was higher than in the case of IMD knockdown (Fig. 8D). The knockdown of Caspar resulted in slightly higher levels of mRNA of some AMP genes than in the malE control (Figs 3 to 7). Nevertheless, it did not increase the resistance to these two bacterial pathogens.

\section{DISCUSSION}

In the present study, we examined the genes coding for potential receptor-proximal components of the IMD pathway in the model beetle T. castaneum, namely, Caspar, $D R E D D$ and $F A D D$, the products of which are constituents of the IMD pathway of Drosophila (Kleino \& Silverman, 2014). We previously reported that the IMD and Toll pathways of $T$. castaneum exhibit a nearly non-specific manner of activation in terms of microbial species used as an elicitor, while the magnitude of activation differed depending on the type of microbe (Yokoi et al., 2012a, b). In the present study, we investigated the involvement of these genes in the activation of this indiscriminate immune pathway, which indicates a marked difference in the immune signaling in this model beetle from the Drosophila system.

Caspar, DREDD and FADD in T. castaneum encode proteins bearing structural features that are typical of the respective family members, including domain configurations, suggesting that these are orthologous genes of the functionally defined counterparts in other organisms. We then showed that these genes were not activated by im- 


\section{A}
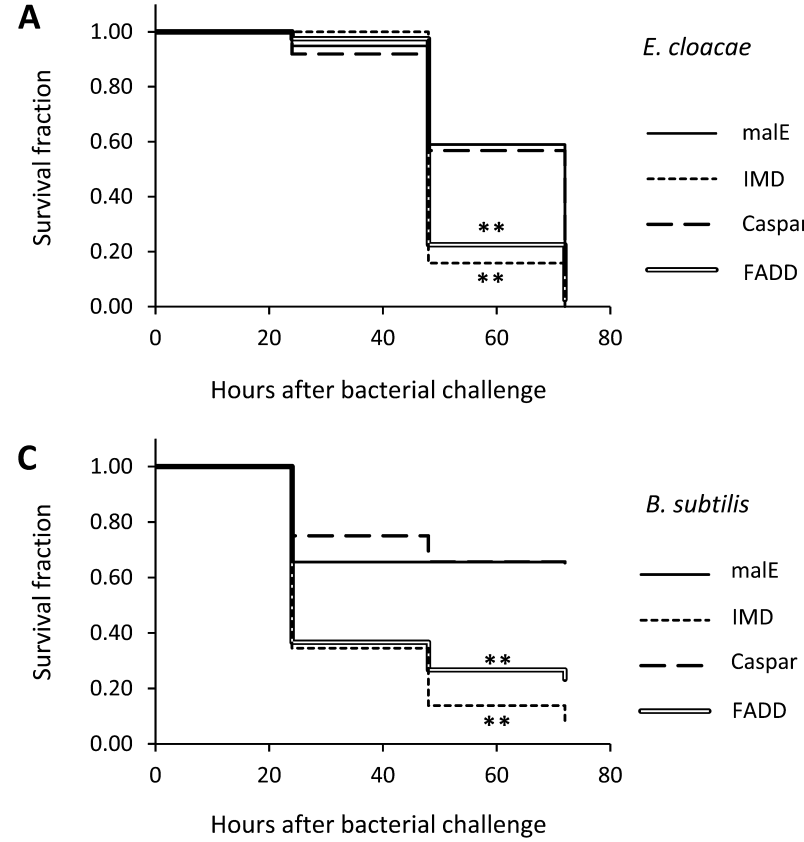

B
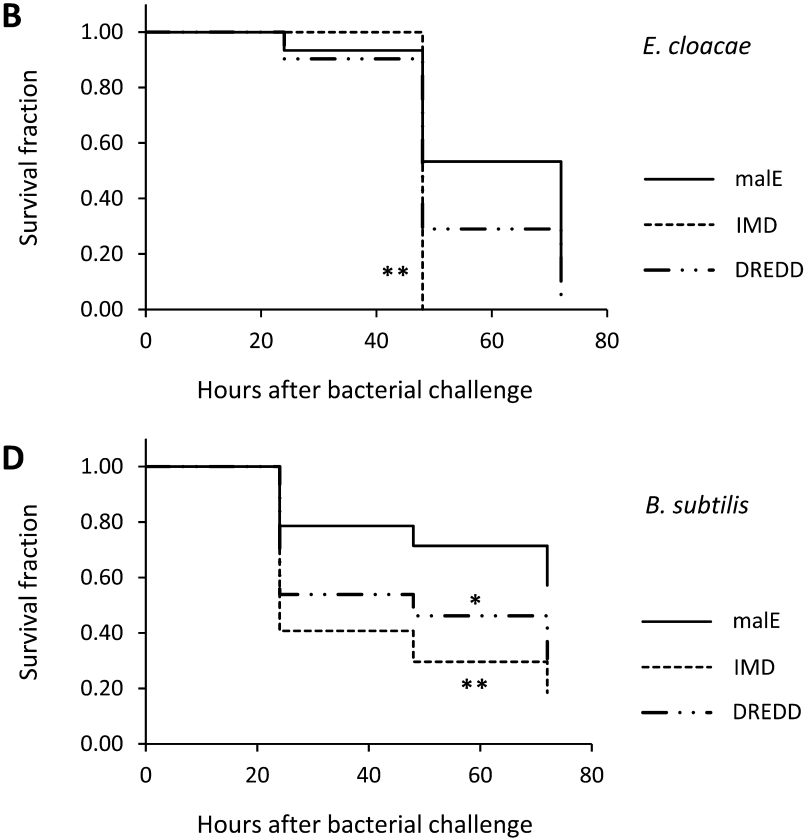

Fig. 8. Effects of the knockdown of Caspar, DREDD and FADD on host defensees against two model bacterial pathogens. Day 3 pupae that were treated with respective preparations of dsRNA on day 0 were challenged by either $E$. cloacae (A and B) or $B$. subtilis (C and $D)$, and the survival of pupae thereafter was monitored and recorded every $24 \mathrm{~h}$. Results are shown in Kaplan-Meier plots. The first set of experiments was carried out using malE, IMD, Caspar and FADD dsRNA-treated pupae (A and C), and the second set using malE, $I M D$, and DREDD dsRNA-treated pupae (B and D). malE dsRNA served as negative controls, while IMD dsRNA provided positive controls. Significant differences from malE-treated controls based on the Gehan-Breslow-Wilcoxon test are denoted by * $(P<0.05)$ or ** $(P<0.01)$.

mune challenges with E. coli, M. luteus or S. cerevisiae. We characterized these genes by estimating their contribution to the microbial induction of two group I genes that represent the IMD pathway read-out. While the results obtained $6 \mathrm{~h}$ after the microbial challenge were obscure, the knockdown of DREDD and FADD resulted in the unequivocal suppression of these two IMD pathway-dependent genes after $24 \mathrm{~h}$. Based on these results, we conclude that DREDD and FADD functioned as canonical constituents of the IMD pathway in this beetle. We previously conducted similar experiments that targeted IMD, and found that the knockdown of IMD took effect by $6 \mathrm{~h}$ after the microbial challenge, with an approximately $50 \%$ reduction in group I gene induction (Yokoi et al., 2012b). Based on similar reductions in the mRNA levels of these factors upon the dsRNA treatment in our present and previous studies, the different phenotypes in the earlier phase of microbial gene induction may be ascribed to the different half-lives of the corresponding proteins. Alternatively, since DREDD encodes the proteolytic enzyme that may process more downstream substrates (Stöven et al., 2000), IMD signaling may be less sensitive to reductions in the amount of the DREDD protein than that of the IMD protein, which provides a molecular scaffold for pathway activation. Moreover, when we assume that the adaptor protein FADD is functioning through its binding to the DREDD protein, the knockdown of FADD showing similar phenotypes to those with the knockdown of DREDD appears to be reasonable (Kleino $\&$ Silverman, 2014). In addition to these assumptions, we cannot rule out the existence of unidentified genes that provide some functional redundancy to DREDD or FADD.
These knockdown phenotypes of DREDD and FADD, and previously obtained IMD knockdown phenotypes in terms of group I gene induction, along with the results of group II and group III genes, appear to be consistent with the results of the survival assays in the present study with the following order of severe impairments in host defenses: IMD knockdown $\geq F A D D$ knockdown $>D R E D D$ knockdown.

We noted that the early-phase responses of the group I genes Att1 and Coll appeared to be slightly more sensitive to the gene knockdown of these intracellular factors when challenged with $E$. coli than with $M$. luteus. In terms of group I gene induction, E. coli is approximately 1.5-fold to 4-fold more potent than M. luteus (the present study, Yokoi et al., 2012b, a). The double knockdown of PGRP-LA plus PGRP-LC, PGRP-LD, or PGRP-LE or the triple knockdown of the PGRP-LA, PGRP-LC and PGRP-LE proteins, however, suppress the induction of group I and II AMP genes by an $E$. coli or $M$. luteus challenge. These findings indicate that PGRP-LA bound by an E. coli-derived DAPtype peptidoglycan or $M$. luteus-derived Lys-type peptidoglycan undergo strong conformational changes that intensively recruit intracellular components, suggesting the lack of a clear difference in the strength of the recognition of both types of peptidoglycans (Koyama et al., 2015). Differences in sensitivities to reductions in group I AMP genes may be attributed to differences in signal strengths, which activate the IMD and Toll pathways during the $E$. coli and $M$. luteus challenges; $M$. luteus may activate the Toll pathway more strongly than $E$. coli.

Group I gene induction by heat-killed $S$. cerevisiae $6 \mathrm{~h}$ after the challenge was not affected by the knockdown of 
Caspar, DREDD or FADD. Twenty-four hours after the challenge, mRNA levels of Att1 in Caspar, DREDD and $F A D D$ knockdown pupae were lower than in control pupae, while Coll expression was not affected by any of the three knockdowns. The induction of group I genes, including Attl by heat-killed $S$. cerevisiae cells, has ceased by this time (Yokoi et al., 2012a). Actually, Att1 mRNA levels in control pupae $24 \mathrm{~h}$ post challenges (approximately 0.05 in Fig. 3F) were not so different from in unchallenged pupae (approximately 0.01 (Yokoi et al., 2012a)) compared to that in the other conditioned pupae. (Att1 levels in control pupae $24 \mathrm{~h}$ post $E$. coli and $M$. luteus challenges was approximately 0.6 and 0.4 respectively). These results indicate that the observed differences reflect variations in the status of the final phase. Furthermore, based on these results, we conclude that DREDD and FADD constitute the canonical IMD pathway of T. castaneum, as in Drosophila, and are not mainly involved in immune signaling induced by the $S$. cerevisiae challenge. Regarding Caspar, its inhibitory effects on the activation of the IMD pathway appeared to be weak or negative and counteracted DREDD in some cases.

The kinetics of group II gene Def3 differed from those of the group I gene upon gene knockdown of these intracellular factors. The suppressive effects of the knockdown of DREDD and FADD became apparent $6 \mathrm{~h}$ after an E. coli or $M$. luteus challenge. These results indicate that the group II gene is more sensitive to a decrease in IMD pathway components than group I genes, which is consistent with our previous findings on IMD (Yokoi et al., 2012b) PGRPLA (Koyama et al., 2015). As described above, PGRP-LA functions as the canonical receptor of the T. castaneum IMD pathway, and this isoform recognizes Gram-negative DAP-type peptidoglycans and Gram-positive Lys-type peptidoglycans. In addition, analyses of the 3' regions of these AMP genes reveal that each group I gene has a single copy of the $\kappa \mathrm{B}$ motif potentially bound by Relish, while group II Def3 has two inverted copies of potential $\kappa \mathrm{B}$ motifs (Yokoi et al., 2012a). Collectively, these findings indicate that the expression of group I genes is readily driven by one Relish molecule, while other classes of transcription factors, such as tissue- or developmental stage-specific factors, synergistically support their high levels of expression (Rus et al., 2013). On the other hand, the full activation of group II Def3 may at least require the occupation of two copies of $\kappa \mathrm{B}$ sites in its proximal promoter region by two molecules of the activated Relish protein. These findings account for the nature of Def 3 , in that it responds more sensitively to the decline in IMD pathway constituents. The degree of Def3 suppression upon knockdown of DREDD and FADD $24 \mathrm{~h}$ after the E. coli challenge appeared to be less conspicuous than those for group I genes and the results of the $M$. luteus or S. cerevisiae challenge after $24 \mathrm{~h}$ was not considered because the induction of Def 3 had ceased before then and also because lower levels of expression of $\operatorname{Def} 3$ were recorded in control pupae post challenge $24 \mathrm{~h}$ with $M$. luteus and $S$. cerevisiae than in the conditioned pupae as described in the previous paragraph
(Yokoi et al., 2012a). This finding may be attributed to the Toll pathway-dependent nature of Def3, which supports the later phase of the induction of $D e f 3$.

The group III genes, $\mathrm{Cec} 2$ and $\mathrm{Cec} 3$, are largely dependent on the Toll pathway for their microbial induction (Yokoi et al., 2012a, b). We determined the involvement of $D R E D D$ and FADD in group III gene regulation and found that the knockdown of DREDD significantly suppressed gene induction $6 \mathrm{~h}$ after the $M$. luteus challenge, while the effects of the knockdown of FADD in terms of the suppression of induction by $E$. coli were recorded $24 \mathrm{~h}$ after the challenge. Since this was the case for $\mathrm{Cec} 2$ and $\mathrm{Cec} 3$, it is reasonable to speculate that these factors function at points common to the regulatory cascade of these group III genes, namely, the Toll pathway, and not due to a decrease in the terminal NF- $\kappa$ B transcription factor of the IMD pathway, Relish. The low sensitivity of group III genes to the knockdown of Relish provides support for this idea (Yokoi et al., 2012a). Six hours after the M. luteus challenge, the knockdown of FADD had a similar outcome to that of DREDD on the induction of both $C e c 2$ and $C e c 3$, indicating that these two Toll-dependent group III genes are also regulated by $F A D D$ and $D R E D D$ via common mechanisms and that the products of these two genes cooperate in this putative path similar to the multimeric molecular assembly activation of the IMD pathway (Kleino \& Silverman, 2014). Since the knockdown of IMD did not suppress the induction of the group III gene by M. luteus after $6 \mathrm{~h}$ (Yokoi et al., 2012b), DREDD and FADD appear to function independently of the IMD protein under these conditions. Twenty-four hours after the $E$. coli challenge, the knockdown of DREDD and FADD resulted in distinct phenotypes; only the knockdown of FADD negatively affected the induction of the group III gene. This result indicates that the FADD protein has different roles under these two distinct conditions.

Caspar was initially identified as a suppressor of IMD signaling based on the genetic screening of a set of Drosophila mutants (Kim et al., 2006). Caspar, which is homologous to mammalian Fas-associating factor 1 (Chu et al., 1995), targets the proteolytic activation of Relish by DREDD. Its loss-of-function phenotype involves the constitutive expression of AMP genes associated with the IMD pathway and increased resistance to Gram-negative infection. In the present study on T. castaneum, the knockdown of Caspar generally resulted in an opposite effect on the induction of the microbial AMP gene when that of DREDD had a negative effect. This result appears to support the functions of DREDD recorded in the present study, which depends on its caspase activity. The basal levels of mRNA of AMP genes were not elevated in unchallenged Caspar knockdown pupae (data not shown). This result, taken together with the mild phenotypes of challenged Caspar knockdown pupae for the induction of the AMP gene, appear to be consistent with the results of survival assays showing that the knockdown of Caspar did not increase the percentage survival over that of malE-treated controls.

Of the three microbes used as elicitors in the present study, we did not obtain clear results with $S$. cerevisiae. 
The transcriptional activation of $T$. castaneum AMP genes by $S$. cerevisiae is very dependent on Toll signaling, even for group I genes (Yokoi et al., 2012b), and in a subsequent study on NF- $\kappa \mathrm{B}$ transcription factor genes involved in the immune challenge from $S$. cerevisiae they may evoke unidentified activating signals other than IMD or Toll signaling (Yokoi et al., 2012a). Therefore, we attribute the equivocal results obtained with $S$. cerevisiae in the present study to the nature of $S$. cerevisiae as an elicitor.

We compared the immune reactions related to IMD, Toll pathway and induction of the AMP gene with our results on $T$. castaneum and previous findings on $D$. melanogaster. Data recorded for T. castaneum were from pupae, whereas those for D. melanogaster were mainly for adult flies. In D. melanogaster, the induction levels of several AMP genes, including Dipetericin, CecropinA, Drosocin and At$\operatorname{tacin} A$, differ in third instar larvae and adults (Verma \& Tapadia, 2012). Therefore, the levels of induction of AMP may differ between pupae and other life stages in T. castaneum. Moreover, comparison of the present results for T. castaneum pupae with previous findings on Drosophila, which were mainly obtained from adults, may be inappropriate. Although the levels of induction of AMP may differ between life stages, both in $D$. melanogaster and $T$. castaneum, the basic immune regulation of AMP genes by Toll and IMD pathways did not markedly differ between the life stages. Therefore, a discussion on the differences in immune signaling via the Toll and IMD pathways between T. castameum and D. melanogaster based on comparisons of the present results with previous findings on D. melanogaster, as described above, is appropriate for the interpretation of immune signaling.

In the present study, we determined the involvement of the Caspar, DREDD and FADD genes of T. castaneum in two immune signaling pathways, namely, the IMD and Toll pathways. The results indicate that the intracellular factors encoded by these genes represented the canonical constituents of the IMD pathway in this beetle, as in Drosophila. Furthermore, the present study demonstrated that DREDD and FADD influenced the activation of Tolldependent group III AMP genes, presumably through their IMD-independent functions to some extent. This intracellular component shared by the two immune signaling pathways provides possible points of signal crosstalk between the two pathways and will facilitate further research on the molecular background of the indiscriminate microbial pathway activation characteristics of $T$. castaneum's antimicrobial immunity.

ACKNOWLEDGEMENTS. This work was supported by JSPS KAKENHI Grant Numbers JP21H03831 to K. Yokoi and 20K06050 to K. Miura. We also thank Y. Yagi, T. Ushimaru, A. Miyanoshita, and M. Murata for providing research material.

\section{REFERENCES}

Blum M., Chang H.Y., Chuguransky S., Grego T., Kandasaamy S., Mitchell A., Nuka G., Lafosse-Paysan T. et al. 2021: The InterPro protein families and domains database: 20 years on. — Nucleic Acids. Res. 49(D1): D344-D354.
Choe K.M., Werner T., Stoven S., Hultmark D. \& Anderson K.V. 2002: Requirement for a peptidoglycan recognition protein (PGRP) in relish activation and antibacterial immune responses in Drosophila. - Science 296: 359-362.

Choe K.M., Lee H. \& Anderson K.V. 2005: Drosophila peptidoglycan recognition protein LC (PGRP-LC) acts as a signaltransducing innate immune receptor. - Proc. Natl. Acad. Sci. U.S.A. 102: 1122-1126.

Chu K., Niu X. \& Williams L.T. 1995: A Fas-associated protein factor, FAF1, potentiates Fas-mediated apoptosis. - Proc. Natl. Acad. Sci. U.S.A. 92: 11894-11898.

De Gregorio E., Spellman P.T., Rubin G.M. \& Lemaitre B. 2001: Genome-wide analysis of the Drosophila immune response by using oligonucleotide microarrays. - Proc. Natl. Acad. Sci. U.S.A. 98: $12590-12595$.

De Gregorio E., Spellman P.T., Tzou P., Rubin G.M. \& LemaiTRE B. 2002: The Toll and Imd pathways are the major regulators of the immune response in Drosophila. - EMBO J. 21: 2568-2579.

Ferrandon D., Imler J.-L., Hetru C. \& Hoffmann J.A. 2007: The Drosophila systemic immune response: sensing and signaling during bacterial and fungal infections. - Nat. Rev. Immunol. 7: $862-874$.

Gangloff M., Murali A., Xiong J., Arnot C.J., Weber A.N., SANDercock A.M., Robinson C.V., SARisky R. et AL. 2008: Structural insight into the mechanism of activation of the Toll receptor by the dimeric ligand Spätzle. - J. Biol. Chem. 283: 14629-14635.

Gobert V., Gottar M., Matskevich A.A., Rutschmann S., Royet J., Belvin M., Hoffmann J.A. \& Ferrandon D. 2003: Dual activation of the Drosophila Toll pathway by two pattern recognition receptors. - Science 302: 2126-2130.

Gottar M., Gobert V., Matskevich A.A., Reichhart J.-M., Wang C., Butt T.M., Belvin M., Hoffmann J.A. \& Ferrandon D. 2006: Dual detection of fungal infections in Drosophila via recognition of glucans and sensing of virulence factors. - Cell 127: $1425-1437$.

Guntermann S. \& Foley E. 2011: The protein Dredd is an essential component of the c-Jun N-terminal kinase pathway in the Drosophila immune response. $-J$. Biol. Chem. 286: 30284-30294.

He Z., Wang P., Shi H., Si F., Hao Y. \& Chen B. 2013: Fas-associated factor 1 plays a negative regulatory role in the antibacterial immunity of Locusta migratoria. — Insect Mol. Biol. 22: 389-398.

Horng T. \& Medzhitov R. 2001: Drosophila MyD88 is an adapter in the Toll signaling pathway. - Proc. Natl. Acad. Sci. U.S.A. 98: $12654-12658$.

Hu S. \& YANG X. 2000: dFADD, a novel death domain-containing adapter protein for the Drosophila caspase DREDD. $-J$. Biol. Chem. 40: 30761-30764.

Hultmark D. 2003: Drosophila immunity: paths and patterns. Curr. Opin. Immunol. 15: 12-19.

Kaneko T., Yano T., Aggarwal K., Lim J.H., Ueda K., Oshima Y., Peach C., Erturk-Hasdemir D. et Al. 2006: PGRP-LC and PGRP-LE have essential yet distinct functions in the Drosophila immune response to monomeric DAP-type peptidoglycan. - Nat. Immunol. 7: 715-723.

Kim M., Lee J.H., Lee S.Y., Kim E. \& Chung J. 2006: Caspar, a suppressor of antibacterial immunity in Drosophila. - Proc. Natl. Acad. Sci. U.S.A. 103: 16358-16363.

Kleino A. \& Silverman N. 2014: The Drosophila IMD pathway in the activation of the humoral immune response. - Dev. Comp. Immunol. 42: 25-35. 
Koyama H., Kato D., Minakuchi C., Tanaka T., Yokoi K. \& MiURA K. 2015: Peptidoglycan recognition protein genes and their roles in the innate immune pathways of the red flour beetle, Tribolium castaneum. - J. Invertebr. Pathol. 132: 86-100.

Kumar S., Stecher G., Li M., Knyaz C. \& Tamura K. 2018: MEGA X: Molecular Evolutionary Genetics Analysis across computing platforms. - Mol. Biol. Evol. 35: 1547-1549.

Lemaitre B. \& Hoffmann J. 2007: The host defense of Drosophila melanogaster. - Annu. Rev. Immunol. 25: 697-743.

Leulier F., Rodriguez A., Khush R.S., Abrams J.M. \& Lemaitre B. 2000: The Drosophila caspase Dredd is required to resist Gram-negative bacterial infection. - EMBO Rep. 1: 353-358.

Meinander A., Runchel C., Tenev T., Chen L., Kim C.H., Ribeiro P.S., BRoemer M., Leulier F. ET AL. 2012: Ubiquitylation of the initiator caspase DREDD is required for innate immune signaling. - EMBO J. 31: 2770-2783.

Naitza S., Rosse C., Kappler C., Georgel P., Belvin M., Gubb D., Camonis J., Hoffmann J.A. Et AL. 2002: The Drosophila immune defense against gram-negative infection requires the death protein dFADD. - Immunity 17: 575-581.

Nassar N., Horn G., Herrmann C.A., Scherer A., McCormik F. \& Wittingghofer A. 1995: The $2.2 \AA$ crystal structure of the Ras-binding domain of the serine/threonine kinase c-Raf1 in complex with RaplA and a GTP analogue. - Nature 375: 554-560.

Paquette N., Broemer M., Aggarwal K., Chen L., Husson M., Erturk-Hasdemir D., Reichhart J.-M., Meier P. et Al. 2010: Caspase-mediated cleavage, IAP binding, and ubiquitination: Linking three mechanisms crucial for Drosophila NF-kappaB signaling. - Mol. Cell 37: 172-182.

Park M.-Y., Jang H.D., Lee S.Y., Lee K.-J. \& Kim E. 2004: Fas-associated factor-1 inhibits nuclear factor-kappaB (NFkappaB) activity by interfering with nuclear translocation of the RelA (p65) subunit of NF-kappaB. - J. Biol. Chem. 4: 2544-2549.

Price M.N., Dehal P.S. \& Arkin A.P. 2009: FastTree: computing large minimum evolution trees with profiles instead of a distance matrix. - Mol. Biol. Evol. 26: 1641-1650.

Rus F., Flatt T., Tong M., Aggarwal K., Okuda K., Kleino A., Yates E., TAtar M. ET AL. 2013: Ecdysone triggered PGRP-LC expression controls Drosophila innate immunity. - EMBO J. 32: $1626-1638$.

Rutschmann S., Jung A.C., Zhou R., Silverman N., Hoffmann J.A. \& FERRANDon D. 2000: Role of Drosophila IKK gamma in a Toll-independent antibacterial immune response. - Nat. Immunol. 1: 342-347.

Silverman N., Zhou R., Stoven S., Pandey N., Hultmark D. \& Maniatis T. 2000: A Drosophila I kappa B kinase complex required for Relish cleavage and antibacterial immunity. - Gen. Dev. 14: 2461-2471.

Silverman N., Zhou R., Erlich R.L., Hunter M., Bernstein E., SCHNEIDER D. \& Maniatis T. 2003: Immune activation of NFkappa B and JNK requires Drosophila TAK1. - J. Biol. Chem. 278: 48928-48934.

Sievers F. \& Higgins D.G. 2021: The Clustal Omega Multiple Alignment Package. - Methods Mol. Biol. 2231: 3-16.

Song E.J., Yim S.H., Kim E., Kim N.S. \& LeE K.J. 2005: Human Fas-associated factor 1, interacting with ubiquitinated proteins and valosin-containing protein, is involved in the ubiquitinproteasome pathway. - Mol. Cell Biol. 25: 2511-2524.

Stöven S., Ando I., Kadalayil L., Engstrom Y. \& Hultmark D. 2000: Activation of the Drosophila NF-kappa B factor Relish by rapid endoproteolytic cleavage. - EMBO Rep. 1: 347-352.

Stöven S., Silverman N., Junell A., Hedengren-Olcott M., Erturk D., Engstrom Y., Maniatis T. \& Hultmark D. 2003:
Caspase-mediated processing of the Drosophila NF-kappa B factor Relish. — Proc. Natl. Acad. Sci. U.S.A. 100: 5991-5996. Sun H.Y., Bristow B.N., Qu G.W. \& Wasserman S.A. 2002: A heterotrimeric death domain complex in Toll signaling. Proc. Natl. Acad. Sci. U.S.A. 99: 12871-12876.

Tomoyasu Y., Miller S.C., Tomita S., Schoppmeier M., GrossMANN D. \& BUCHER G. 2008: Exploring systemic RNA interference in insects: a genome-wide survey for RNAi genes in Tribolium. - Genome Biol. 9: R10, 22 pp.

Tribolium Genome Sequencing Consortium 2008: The genome of the model beetle and pest Tribolium castaneum. - Nature 452: 949-955.

Valanne S., Wang J.H. \& Rämet M. 2011: The Drosophila Toll signaling pathway. - J. Immunol. 186: 649-656.

VERMA P. \& TAPADIA M.G. 2012: Immune response and anti-microbial peptides expression in malpighian tubules of Drosophila melanogaster is under developmental regulation. - PLoS ONE 7(7): e40714, 12 pp.

Weber A.N.R., Tauszig-Delamasure S., Hoffmann J.A., Lelievre E., Gascan H., Ray K.P., Morse M.A., Imler J.L. et al. 2003: Binding of the Drosophila cytokine Spatzle to Toll is direct and establishes signaling. — Nat. Immunol. 4: 794-800.

Wu L.P. \& ANDERSON K.V. 1998: Regulated nuclear import of Rel proteins in the Drosophila immune response. - Nature 392: 93-97.

Yokol K., Koyama H., Ito W., Minakuchi C., Tanaka T. \& Miura K. 2012a: Involvement of NF- $\kappa B$ transcription factors in antimicrobial peptide gene induction in the red flour beetle, Tribolium castaneum. - Dev. Comp. Immunol. 38: 342-351.

Yokoi K., Koyama H., Minakuchi C., Tanaka T. \& Miura K. 2012b: Antimicrobial peptide gene induction, involvement of Toll and IMD pathways and defense against bacteria in the red flour beetle, Tribolium castaneum. - Results Immunol. 2: $72-82$.

Zou Z., Evans J.D., Lu Z., Zhao P., Williams M., Sumathipala N., Hetru C., Hultmark D. et Al. 2007: Comparative genomic analysis of the Tribolium immune system. - Genome Biol. 8: R177.

Received July 19, 2021; revised and accepted December 17, 2021 Published online January 12, 2022

\section{Supplementary figures, supplementary tables and supplementary data}

All supplementary figures, supplementary table and supplementary data were uploaded to figshare. DOI: 10.6084/ m9.figshare.c. 5429688

\section{Supplementary Fig. 1}

Approximately-maximum-likelihood phylogenetic trees of (A) Caspar, (B) DREDD and (C) FADD. Numbers near branch points and along the branches are bootstrap values and lengths of branches, respectively. DOI: 10.6084/m9.figshare.14618898

(A) Phylogenetic tree results using the Caspar genes of $T$. castaneum (Tc-caspar), A. aegypti (Ae-caspar, GenBank ID: XP_021697938.1), A. gambiae (Ag-caspar, GenBank ID: XP 316513), D. melanogaster (Dm-caspar, GenBank ID: NP 611080.1), B. mori (isoform X1) (Bm-Caspar, GenBank ID: XP_037873675.1), A. mellifera (isoform X1) (Am-Caspar, GenBank ID: XP_392750.1), N. lugens (N1-Caspar, GenBank ID: XP_022206141), mouse orthologue FAF1 (Mouse FAF1, GenBank ID: NP_032009.2) and the human orthologue, FAF1 (Human-FAF1, GenBank ID: CAB67705.1). The fasta file of the predicted amino acid sequences is in Supplementary Data 1 (Caspar-AA-seqs.fa), 
(B) Phylogenetic tree results using the DREDD genes of T. castaneum (Tc-Dredd), A. aegypti (Ae-DREDD, GenBank ID: ABI74776.1), A. gambiae (Ag-Dredd, GenBank ID: XP 552560.2), D. melanogaster (Dm-DREDD (isoform gamma), GenBank ID: AAC33118.1), B. mori (Bm-DREDD, GenBank ID: NP_001108337), A. mellifera (Am-DREDD, GenBank ID: XP_006570976), N. lugens (N1-DREDD, GenBank ID: XP_039280806), mouse orthologue Caspase-8 (Mouse-Caspase-8, GenBank ID: NP 032009.2) and the human orthologue, Caspase-8 (Hm-Casp8, GenBank ID: AAD24962.1). The fasta file of the predicted amino acid sequences is in Supplementary Data 1 (DREDD-AA-seqs.fa),

(C) Phylogenetic tree results using the FADD genes of T. castaneum (Tc-FADD), A. aegypti (Ae-FADD, GenBank ID: EAT46931.1), A. gambiae (Ag-FADD, GenBank ID: XP_308597.4), D. melanogaster (Dm-FADD, GenBank ID: AAF55950.1), B. mori (Bm-FADD, GenBank ID: NP_001189465), A. mellifera (Am-FADD, GenBank ID: XP_006572135), mouse FADD (Mouse-FADD, GenBank ID: NP 034305) and human FADD (Hm-FADD, GenBank ID: CAG33019.1). The $N$. lugens orthologue was not found in the $N$. lugens genome. The fasta file of the predicted amino acid sequences is in Supplementary Data 1 (FADD-AA-seqs.fa).

\section{Supplementary Fig. 2}

InterPro scan results of (A) Tc-Caspar, (B) Tc-DREDD and (C) Tc-FADD. The numbers in the upper part of each figure indicate the sequence numbers of amino acid residues counted from the $\mathrm{N}$ terminal end. The gff result file of each amino acid sequence is uploaded in Supplementary Data 3. Detailed results are on the InterPro site (URL: https://www.ebi.ac.uk/interpro/about/interproscan/). DOI: 10.6084/m9.figshare.14625522

\section{Supplementary Table 1}

Primer sequence list in the present study. DOI: 10.6084/ m9.figshare. 14625510

\section{Supplementary Data 1}

Fasta files of predicted amino acid sequences of Caspar, DREDD and FADD used for phylogenetic trees. DOI: 10.6084/ m9.figshare.16918201

\section{Supplementary Data 2}

Aln files (Clustal omega output files) and newick files (FastTree output files) of Caspar, DREDD and FADD. DOI: 10.6084/ m9.figshare. 14618706

\section{Supplementary Data 3}

Gff files of InterProScan results of Tc-Caspar, Tc-DREDD and Tc-FADD. DOI: 10.6084/m9.figshare. 16918210 